\begin{tabular}{|c|c|}
\hline 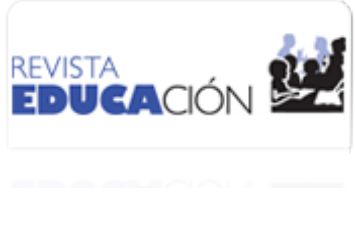 & $\begin{array}{l}\text { Revista Educación } \\
\text { ISSN: 0379-7082 } \\
\text { ISSN: } 2215-2644 \\
\text { revedu@gmail.com } \\
\text { Universidad de Costa Rica } \\
\text { Costa Rica }\end{array}$ \\
\hline
\end{tabular}

\title{
Enrique Moreno y de los Arcos (1943-2004). La vida de un pedagogo auténtico
}

\author{
García Cerda, Pólux Alfredo \\ Enrique Moreno y de los Arcos (1943-2004). La vida de un pedagogo auténtico \\ Revista Educación, vol. 45, núm. 1, 2021 \\ Universidad de Costa Rica, Costa Rica \\ Disponible en: http://www.redalyc.org/articulo.oa?id=44064134021 \\ DOI: https://doi.org/10.15517/revedu.v45i1.42289
}

Esta obra está bajo una Licencia Creative Commons Atribución-NoComercial-SinDerivar 3.0 Internacional. 


\section{Enrique Moreno y de los Arcos (1943-2004). La vida de un pedagogo auténtico}

Enrique Moreno y de los Arcos (1943-2004). The life of an Authentic Pedagogue

Pólux Alfredo García Cerda

Universidad Nacional Autónoma de México, México

poluxgarcia@filos.unam.mx
DOI: https://doi.org/10.15517/revedu.v45i1.42289

Redalyc: http://www.redalyc.org/articulo.oa?id=44064134021

Recepción: 26 Junio 2020

Aprobación: 27 Septiembre 2020

\section{Resumen:}

En el presente texto se esboza el primer perfil biográfico de un pensador importante para la pedagogía mexicana. Para su realización, se usó el método histórico de comprensión aplicando las nociones hermenéuticas de texto y narrativa biográfica. La intencionalidad de su vida, su pensamiento y su obra se reconstruyeron a través de una primera localización e interpretación de tres grandes fuentes (su último curriculum vitae, su archivo laboral personal y su obra escrita publicada). La trayectoria intelectual de este pedagogo se desarrolló en instituciones donde diversas tradiciones epistemológicas se disputaron el estatuto disciplinar de la Pedagogía. Uno de esos lugares fue la Universidad Nacional Autónoma de México, lugar donde se dedicó a trabajar la mitad de su vida y en la que aún sigue vivo su legado y sus ideas. Con una personalidad compleja, entre contradicciones y convicciones personales, su aprecio por la cultura en lengua castellana, fue un destacado docente, investigador y difusor de la cultura pedagógica clásica y moderna que espera ser conocido, leído y estudiado más allá del país donde siempre vivió y falleció.

Palabras clave: Pedagogo, Pedagogía clásica, Pedagogía moderna, Historia de la Pedagogía, Investigación histórica.

\section{Abstract:}

This an initial biographical depiction of one of Mexico's foremost pedagogical thinkers which is based on a historical method that applies hermeneutical text and biographical narrative. The purpose of Moreno y de los Arco's' life, thought and work were reconstructed based on three primary sources - his latest resume, personal work archive and published material. The intellectual career of this pedagogue was developed in institutions where various epistemological traditions vied for the disciplinary status of Pedagogy. One of those places was the National Autonomous University of Mexico, a place where he spent half his life working and where his legacy and ideas are still alive. Despite his complex personality, imbued by contradictions, personal convictions and a deep appreciation for Spanish language and culture, de los Arcos was an outstanding teacher, researcher and disseminator of classical and modern pedagogy who deserves to be known, read and studied beyond Mexico, the country where he lived and died. KeYworDs: Pedagogue, Classical Pedagogy, Modern Pedagogy, History of Pedagogy, Historical Research.

\section{INTRODUCCIÓN}

En noviembre de 2015 se realizó el evento Enrique Moreno y de los Arcos. Vida, pensamiento y obra de un pedagogo comprometido, el cual motivó la publicación de los primeros tres estudios de la obra morenista (Peralta, 2015; Delgado, 2017; García, 2017). A continuación, se ofrece un bosquejo biográfico en el que se señala cuáles fueron los logros de una trayectoria académica y profesional, y las razones por las cuales una obra pedagógica compleja debe cruzar las fronteras internacionales para ser leída por pedagogos y educadores más allá del rio Suchiate. El 15 de junio de 1985, el pedagogo mexicano Enrique Moreno y de los Arcos (1943-2004) publicó la tercera entrega de su artículo Orientación. El sistema de orientación integral en el periódico mexicano unomásuno, medio de comunicación masiva donde se había publicado, en 1980, el libro Las batallas en el desierto de José Emilio Pacheco. A diferencia del texto que hoy es considerado clásico para la literatura mexicana y latinoamericana, aquel artículo no tuvo siquiera un impacto similar, a pesar de que se suscitó un hecho insólito en la relación histórica entre la tradición pedagógica mexicana y las tradiciones pedagógicas centroamericanas. Bajo la dirección de importantes orientadores mexicanos, Enrique Moreno realizó una investigación experimental que diseñó, aplicó y evaluó el Inventario de Diagnóstico Diferencial 
(DD-1), el cual evolucionó en el DD-2 y DD-3. Entre 1970 y 1986, fue tan exitosa la tercera versión del instrumento que instituciones educativas mexicanas, como la Universidad Nacional Autónoma de México (UNAM) y el Instituto Politécnico Nacional (IPN), la adoptaron. Todavía más relevante es que el DD-3 también lo adoptó la Organización de Estados Americanos (OEA) y fue tal el peso de su recomendación a los países asociados que se adoptó y aplicó en la Universidad de San Carlos (Guatemala) y el sistema de orientación de Nicaragua (Moreno, 1993). Recientemente, el autor del presente artículo ha concluido una investigación intitulada El pensamiento de Enrique Moreno y de los Arcos y su idea de plan de estudios, la cual se realizó en el Doctorado en Pedagogía de la Facultad de Filosofía y Letras (FFyL-UNAM). En una de las reflexiones finales, se afirmó la necesidad de estudiar, valorar y difundir críticamente el significado de las ideas de Enrique Moreno y su legado intelectual para la pedagogía latinoamericana.

\section{Metodología}

Para la realización de este bosquejo biográfico, se empleó el método histórico de comprensión. Perteneciente al paradigma historicista, se sostiene que el sentido de una idea puede captarse si se interpreta el tipo histórico que la pensó y la concepción de mundo en que surgió (Larroyo, 1979; Luzuriaga, 1980). El primero es la asimilación de un ideal de ser humano, y el segundo es el conjunto de circunstancias históricas propias de la vida de una persona. Por su parentesco metodológico, se actualizó el método originario de la Historia de las Ideas Pedagógicas a través de la noción de texto perteneciente también a la tradición aristotélica (Ursúa, Ayestarán y González, 2004). En términos hermenéuticos, el texto se puede clasificar en tres tipos, uno escrito, uno oral y otro como acción significativa. Enseguida, se retoma la definición de texto como discurso fijado por la escritura y que ostenta sentido y referencia, "sentido, en cuanto susceptible de ser entendido o comprendido por el que lo lee o lo ve o lo escucha; referencia, en cuanto apunta a un mundo, sea real o ficticio, indicado o producido por el texto mismo" (Beuchot, 2005, pp. 34-35).

La actualización de las nociones de tipo histórico como trayectoria vital, desde una perspectiva biográfica, y concepción de mundo, como entramado de problemas y situaciones en los cuales un pensamiento intermedia, se basó en una reconstrucción histórica de la vida de un importante catedrático universitario según tres fuentes: 1) su último Curriculum Vitae (2004), el cual fue proporcionado por una profesora del Colegio de Pedagogía (UNAM) que fue su asistente personal por más de veinte años; 2) su archivo laboral personal, con número de expediente 37397 y el rótulo de MOAE431115 (la carpeta se ubicó en el archivo histórico de la Dirección General de Personal, UNAM); y 3) testimonios recuperados y que estaban diseminados en escritos de Enrique Moreno y de colegas cercanos, como la entrevista que le realizó la Dra. María Esther Aguirre Lora (investigadora adscrita al Instituto de investigaciones Sobre la Universidad y la Educación, UNAM). Desde un diálogo de saberes pedagógicos y hermenéuticos, se interpretó biográficamente la intencionalidad plural de una trayectoria vital situada como una expresión sintética de experiencias, cuyo devenir se materializó en intereses, perspectivas y representaciones personales (Escamilla y Rodríguez, 2010). Bajo este marco, se tramó historiográficamente el relato biográfico de un experto en temas educativos que leía literatura universal en novelistas como Charles Dickens, aunque le fascinaba también la literatura mexicana en poetas como Rubén Navarro. En ese sentido, ¿quién fue aquel pedagogo que, después de dar clases, gustaba fumar Raleigh (una antigua marca de cigarros) y se atrevía a señalar, en tono sutilmente irónico, de charlatán a un colega en la institución donde cotidianamente trabajaba?

\section{Resultados}

Enrique Moreno y de los Arcos nació en la capital mexicana un 15 de noviembre de 1943, en una familia de limitados recursos económicos. Aquella década de 1940 fue clave para México, pues acaeció el llamado 
viraje conservador, donde se dejaba atrás el populismo nacionalista del Gral. Lázaro Cárdenas y se consolidaba el proyecto de Unidad Nacional del Gral. Manuel Ávila Camacho. Tal era la conversión de un mundo mayormente rural a uno urbanizado y orientado político-económicamente hacia los Estados Unidos de América. Su padre fue Roberto Moreno y García (1905-1973), quien era recordado así por uno de sus hijos:

Mi padre, además de pedagogo con anterioridad había hecho la carrera de economía, y era muy aficionado a la historia. La carrera de economía la estudió porque en aquel entonces militaba en el Partido Comunista y era fundamental que estudiaran esta materia. La historia era un componente adicional, pero importante. Como pedagogo que era, mi padre fue el introductor de los métodos audiovisuales en las escuelas de México y también fue, entre otras cosas, de los fundadores del Fondo de Cultura Económica. (...) Mi padre era de escasos recursos y sobrevivimos, mal que bien; con tenacidad de mi madre se logró comprar una casa en la colonia llamada Unidad Modelo. ${ }^{[1]}$ (...) Era una unidad habitacional que se hizo para profesores. Y un día, cuando yo estaba en $4^{\circ}$ de primaria dije, ahí, durante la comida con mis padres: 'la única materia que no me interesa es la historia'. Entonces mi padre, que era un hombre realmente interesante y muy culto, dijo: 'espérate, desde el próximo lunes vamos a hablar en las tardes de historia'. Y así mi padre armó un curso de historia de Grecia y de Roma, con diapositivas y todo, y en ese curso nos cambió la vida a mi hermano gemelo y a mí, A mí me fincó la vocación por la historia y a mi hermano la vocación por la pedagogía (Olivera, Rueda y Espejel, 1998, p. 229).

Como egresado de la Escuela Normal de Maestros, Moreno y García fue reconocido en su tiempo por altos mandos de la educación mexicana que lo contactaron para trabajar después en la Organización de las Naciones Unidas para la Educación, la Ciencia y la Cultura (UNESCO). Participó en la fundación de la editorial Fondo de Cultura Económica (FCE). Afín al anarquismo latinoamericanista, Roberto Moreno conoció a intelectuales como el pedagogo uruguayo Jesualdo Sosa y el poeta cubano Nicolás Guillén. Su activismo político fue frenado de golpe al notificarle la expulsión del Partido Comunista Mexicano (PCM). El motivo: haber publicado un libro sin permiso del partido. Después de este percance, fue catedrático del Colegio de Pedagogía (UNAM), inspector de enseñanza Normal y especialista en analfabetismo y enseñanza audiovisual. (Aguirre, 1998). El fundador del FCE, Daniel Cosío Villegas, fue pieza clave para recibir al contingente de españoles refugiados durante el gobierno cardenista. Una de esas refugiadas fue su esposa Adela de los Arcos, originaria de Galicia y que trabajó por más de treinta años en la docencia de educación primaria. Este matrimonio limitado en recursos económicos, pero rico en cuanto a capital cultural, tuvo gemelos, uno fue Enrique y el otro fue Roberto (1943-1995); éste último, famoso historiador y bibliógrafo especializado en Historia de la Ciencia, Mundo náhuatl antiguo e Historia de la Nueva España. Siguiendo los pasos de su padre, Enrique Moreno estudió en la Escuela Normal de Maestros, ejerció como docente de primaria (igual que su madre):

Me tocó vivir la historia de (...) [la escuela] 'Bartolomé Cossío’ porque llegaron originalmente el maestro Tapia y Chela Graciela González de Tapia- a fundar una escuela experimental en la colonia el Retoño, [ubicada cerca del domicilio de Moreno] y cuando salimos mi hermano y yo de la Normal, como teníamos alto promedio, nos dieron a escoger escuela y entonces elegimos la misma escuela en la que trabajaba mi madre (Aguirre, 1998, p. 183).

El director de aquella escuela experimental fue José de Tapia (1896-1989), pedagogo español, anarquista y discípulo de Celestin Freinet. Su paso como profesor de primaria duró sólo dos años, porque decidió cursar la Licenciatura en la FFyL-UNAM, si bien, más tarde se graduó también de la Maestría y el Doctorado en Pedagogía en esa institución. En el caso de la primera, abierta desde 1960, se integró a la tercera generación de pedagogos y recibieron cursos nunca antes impartidos en México: Didáctica General, Historia de los sistemas de segunda enseñanza, Fundamentos Biológicos de la Educación, Conocimiento de la Adolescencia, Antropometría pedagógica, Orientación Vocacional y Profesional, Corrientes de la psicología contemporánea, Teoría pedagógica, Historia de la educación en México, Conocimiento de la Infancia, Conocimiento de la Adolescencia, Psicopatología de la Adolescencia, Psicotécnica pedagógica, Educación audiovisual, Didáctica de las Lenguas Vivas, Fundamentos sociológicos de la Educación, Organización escolar, Técnica de la Investigación pedagógica, Filosofía de la educación, Historia de los Sistemas de Enseñanza Normal, Historia de las Universidades, Seminario de Didáctica y Organización, Historia General 
de la Educación, Teoría pedagógica, Pedagogía comparada y Seminario de Textos Clásicos de la Pedagogía. Los maestros que más incidieron en su formación fueron los pedagogos transterrados Domingo Tirado Benedí ${ }^{[2]}$ (1898-1971) y Santiago Hernández Ruiz[3] (1901-1988) junto con el filósofo y educador Francisco Larroyo[4] (1912-1981), éste último fue fundador del Colegio de Pedagogía (UNAM). A través de sus enseñanzas, Moreno recibió las ideas de pensadores hispanoparlantes vinculados a la educación como el filósofo español José Ortega y Gasset, el filósofo mexicano José Vasconcelos y el pedagogo español Lorenzo Luzuriaga. Debido a su labor como orientador educativo, estuvo modestamente conectado con la educación centroamericana y uno de los móviles fue la idea de formación integral que retomó del filósofo español:

Conforme se ascienden los peldaños del sistema escolar, más especializada y concreta se encuentra la enseñanza de diferentes disciplinas. La preocupación por lograr una mejor capacitación científica y profesional de los egresados de las instituciones de educación superior hace relegar frecuentemente su educación en sentido lato, esto es, el proceso de formación que convierte a los individuos en activos participantes de su cultura y de su vida colectiva. Son producidos, entonces, los profesionistas que Ortega y Gasset llamó los ‘nuevos bárbaros', personas con profundo conocimiento de su disciplina pero totalmente ajenas a otras creaciones humanas tanto en lo científico y lo filosófico, como en lo artístico (Moreno, 1993, p. 172).

Con una formación abierta a las Ciencias y Humanidades, obtuvo su primer trabajo, en 1967: el cargo de psicotécnico al interior de la Dirección General de Orientación y Servicios Sociales (DGOSS-UNAM). Sin embargo, poco después comenzó su carrera docente, la cual ejerció durante 36 años en la UNAM e instituciones como la Universidad Iberoamericana, Universidad Veracruzana y Universidad Autónoma de Nuevo León. Además de la formación pedagógica, se especializó en siete campos disciplinares: Teoría pedagógica y Didáctica, Epistemología, Pedagogía experimental, Historia de la educación y de la pedagogía, Orientación educativa, Bibliografía pedagógica y Humor pedagógico. Desde la década de 1960 hasta unos años antes de su fallecimiento en 2004, su especialización en aquellos campos se plasmó en distintos artículos publicados por revistas como PEDAGOGÍA y Paedagogium. Revista Mexicana de Educación y Desarrollo, ésta última fundada por él y su discípulo Benito Guillén. Ambas revistas hoy están extintas. A continuación, se indican sus publicaciones más sobresalientes:

- La educación del adolescente nábuatl (I), PEDAGOGÍA, Núm. 1, sep-oct, 1965.

- Comparación entre las leyes fundamentales de educación de México, Alemania Occidental y Alemania Oriental, PEDAGOGÍA, Núm. 3, mar-abr, 1966.

- Los orígenes de la pedagogía en México, Enseñanza más aprendizaje. Revista de la Escuela Normal Superior de Nuevo León, No. 5, Sep. 1982.

- El lenguaje de la pedagogía, Omnia, Vol. 2, núm. 5, 1986.

- La crisis de la pedagogía, Paedagogium, Núm. 11, 2002.

El grueso de su obra se publicó en libros determinantes para la denominada polémica sobre el estatuto disciplinar de la Pedagogía, es decir, la polémica Pedagogía vs Ciencias de la educación.

- Hacia una teoría pedagógica', México, UNAM, 1999.

- Pedagogía y Ciencias de la educación', México, UNAM, 1990.

- Examen de una polémica 'en relación al' examen, México, UNAM, 1996.

- Los paradigmas metodológicos de la investigación pedagógica, México, UNAM, 1990.

- Plan de estudios y 'Curriculum', México, UNAM, 1990.

- Principios de pedagogía asistemática. Ensayos, México, UNAM, 1993.

Toda su obra escrita puede entenderse como una defensa de la Pedagogía, sustentada epistemológicamente en la tradición clásica, pero compuesta de cuatro vertientes totalmente válidas: pedagogía teórica, pedagogía práctica, pedagogía técnica y pedagogía tecnología. Moreno (1993) pensaba que la pedagogía se convertía en ciencia sólo bajo la vertiente técnica. Este juicio cae en el cientificismo, porque revela una inclinación desmedida por la pedagogía experimental y un desconocimiento de las demás vertientes, sobre todo la teórica 
que actualmente ha impulsado a la pedagogía a explorar nuevos horizontes disciplinares como la Sociología Crítica de la educación y la Hermenéutica.

En dicha tradición se encontraban autores y obras que consideró clásicos, es decir, relevantes para la constitución de la pedagogía como disciplina autónoma: Plutarco y La educación de los niños, Clemente de Alejandría y El Pedagogo, Francis Bacon y sus Escritos pedagógicos, Diderot y su Plan de una universidadpara el gobierno de Rusia, John Locke y sus Pensamientos sobre educación, Immanuel Kant y su Pedagogía, Herbart y su Pedagogía general derivada del fin de la educación, Natorp y su Curso de pedagogía social, Meumann y su Pedagogía experimental, (Moreno, 1993). A la par de estos clásicos universales, Moreno impulsó el estudio y valoración de la pedagogía mexicana representada en el Tratado elemental de pedagogía Manuel Flores y el Tratado elemental de pedagogía de Luis Ruiz (Moreno, 1993).

$\mathrm{Al}$ menos cuatro de esos títulos (los de Plutarco y Bacon, de la primera categoría, y los de Flores y Ruiz, de la segunda) fueron publicados en México gracias a su proyecto editorial que llamó Biblioteca Pedagógica con apoyo de la UNAM. Este proyecto se distingue por dos detalles: 1) el proyecto tuvo lugar a la par de la instauración del neoliberalismo en México (década de 1980), justamente cuando el mercado editorial se contrajo abruptamente por la crisis económica y se optó por publicar predilectamente literatura pedagógica y educativa de corte psicologicista, sociologicista y tecnicista; 2) el proyecto fue el último que publicó intencionadamente textos clásicos de la pedagogía (en las dos categorías mencionadas), pues Moreno tomó como modelo a la Biblioteca pedagógica que Luzuriaga dirigió en España con Ediciones La Lectura y luego, ya en Argentina, con la Editorial Losada.

El pensamiento que impulsó este proyecto, y defendía la pedagogía de quienes querían desaparecerla, supeditarla o sustituirla por las Ciencias de la Educación, formuló una teoría que él denominó la Polémica de los modelos pedagógicos (Moreno, 1999). Moreno erró al denominar pedagógica una polémica que, por referirse a paradigmas de enseñanza y aprendizaje (y no a una reflexión sistemática sobre la pedagogía como disciplina), debe ser entendida como la polémica de los modelos didácticos. Esta teoría fue innovadora, pues usó fuentes literarias (como la novela Oliver Twist, de Charles Dickens) para demostrar hipótesis científicas:

Parecería existir en la enseñanza profesional y de las actividades prácticas un conflicto entre modelos pedagógicos susceptible de ser rastreado de muy atrás. Dejando de lado un intento de explicación histórica que contemple las necesarias implicaciones sociales y económicas, y prestando atención solamente a la posible validez de la distinción propuesta, podemos afirmar que existe un doble enfoque pedagógico para la enseñanza, que designaremos como los modelos escolar y artesanal de la educación. Podrían encontrarse relaciones del primero con la enseñanza de la enseñanza de los sofistas, las escuelas de los retóricos, la universidad napoleónica y nuestras modernas escuelas y facultades. El segundo podría relacionarse con las escuelas de Platón y Aristóteles, los escritos de Cicerón, Séneca y Tácito, la universidad según Humboldt y nuestros institutos de investigación (Moreno, 1999, p. 21).

Entre las décadas de 1980 y 2000, la polémica de los modelos ofrecía una hipótesis interesante sobre la presencia de aquellos científicos de la educación que pretendían ser reconocidos en el medio académico como pedagogos, pero que solicitaban, irónicamente, su desaparición de los estudios universitarios. A estos sujetos, Moreno les llamó charlatanes y hacían tanto daño a la comunidad universitaria como aquellos que se hacen pasar por médicos, portadores del nefasto mensaje que enferman en lugar que curar los males sociales. Entendida la autenticidad de su posicionamiento, así caracterizó cada modelo en la teoría pedagógica que le valió el título de Doctor en Pedagogía, en 1996:

El modelo artesanal (...) es la forma natural de enseñanza que ha acompañado al hombre desde su aparición como tal. Es esencialmente mimético, a diferencia del modelo escolar que podemos considerar (...) lúdico. En efecto, la enseñanza artesanal confía el aprendizaje a la imitación del ejemplo que proporciona el maestro en el desarrollo de la actividad. En la escuela, en cambio, las actividades se desarrollan como si fueran reales, no siéndolo, sin embargo. (...) La verdadera originalidad de la escuela podríamos decir que radica en los siguientes aspectos: sistematiza los contenidos de la enseñanza, busca fundamento a lo que se ha de enseñar y produce técnicas y materiales para lograr el aprendizaje (Moreno, 1999, pp. $55,75)$. 
En tiempos de la crítica a la escuela (Illich, 1973; Carnoy, 1977), Moreno defendió la escuela, en específico la escuela pública, pues representaba una de las invenciones socialmente más originales y que había dado lugar a grandes científicos y humanistas como Tommaso Campanella, Charles Rollin y Juan Amós Comenio (Moreno, 1999). Teniendo por misión resguardar y estudiar la escuela, de la Pedagogía surgieron cuatro vertientes disciplinares que Moreno reconoció como válidas. La crisis de la escuela y la crisis de la pedagogía, se debían, en gran medida, a la polémica entre Pedagogía y Ciencias de la educación:

Gaston Mialaret, ${ }^{[5]}$ que después de haber publicado varios libros sobre pedagogía, de iniciación a la pedagogía, de pedagogía experimental, (...) de repente alborozadamente nos dice que se acabó la pedagogía y que de aquí en adelante se llama atinadamente Ciencias de la Educación. Y argumenta por qué. Yo creo que todo mundo tiene derecho a cambiar de opinión, lo que no me parece bien es que lo haga tan mal como lo hace (Moreno, 2002, p. 4).

Moreno fue un pedagogo multifacético, cuya vida se cifró como una respuesta al fenómeno que denominó La crisis de la pedagogía, es decir, la generalizada falta de especialistas en temas educativos, sin una adecuada formación histórica, y carentes de un lenguaje profesional necesario para enseñar, investigar y difundir sus ideas. Después de un diagnóstico publicado en un artículo con idéntico título, señaló cuatro de sus causas: 1) facundia irrestricta (entendida como el uso equívoco del lenguaje), 2) preterición histórica (o bien, la escritura presentista de la historia), 3) confusión teórica (o la clasificación vaga de enfoques para el estudio de los fenómenos educativos) y, 4) incuria metodológica (disimulo de fuentes al construir un objeto de estudio). Uno de los síntomas de esta crisis se podía notar en el lenguaje usado para la formación e investigación pedagógica. La sustitución de vocablos clásicos como Plan de estudios, Prueba y Orientación, por anglicismos como Curriculum, Test y Guidance, era una muestra de cómo se estaba perdiendo paulatina e irreparablemente el legado nacional. De la invasión de la tradición anglosajona, gestora de una formulación hegemónica de las Ciencias de la Educación, sucedió la imposición de la cultura norteamericana que pretendió aniquilar la tradición clásica. Quienes fueron sus estudiantes, colegas y amistades vieron en él un pensador comprometido con el presente y un crítico de la formación pedagógica actual, además de hábil para combinar la tradición humanista en exponentes como el arquitecto romano Vitruvio y la tradición científica en exponentes como el filósofo analítico argentino Mario Bunge. Ni sus textos ni sus ideas fueron discutidos o replicados por alguno de los intelectuales que él interpeló en la polémica Pedagogía vs Ciencias de la educación: ni Gaston Mialaret, ni Antonio Santoni, ni Ricardo Sánchez Puentes, ni Alicia de Alba, ni ArgenMex como Alfredo Furlán, ni Ángel Díaz Barriga (Moreno, 1999). Una excepción fueron los epistemólogos de la Universidad Pedagógica Nacional: Monserrat Bartomeu, Fernando Juárez, Santiago Alzueta e Irma Juárez. Ellos defendieron la noción de Ciencia Educativa Crítica desde autores como Wilfred Carr y Stephen Kemmis y criticaron, atinadamente, la tendencia univocista en la predilección de Moreno por la pedagogía experimental (Bartomeu, Juárez, Alzueta y Juárez, 1993).

En tanto, Moreno fue un universitario y, si la misión de la universidad moderna es cumplir tres funciones fundamentales (docencia, investigación y difusión de la cultura), su vida representa el compromiso de un universitario cabal que ejerció como catedrático, investigador -adscrito al Sistema Nacional de Investigadores (SNI) entre 1984 y 2002- y difusor de la cultura, recordando su labor en el periódico unomásuno y su participación en Radio UNAM en el programa Iniciación a la pedagogía en 1987. También fue asesor en el diseño de planes y programas de estudio en instituciones como la Secretaría de Educación Pública y universidades de la capital y el interior de la República Mexicana, al mismo tiempo fue organizador y coordinador de distintos Congresos pedagógicos nacionales en las décadas de 1980 y 1990. Además de haber sido un pedagogo totalmente entregado a los espacios donde laboró, también incursionó como traductor del libro La evaluación de programas educativos de W. Astin y R. Panos. Pero el significado más profundo de su legado se encuentra al interior de la UNAM, donde ejerció cargos administrativos importantes como el de Secretario General de la Facultad de Filosofía y Letras (cuando el Director fue Ricardo Guerra, filósofo 
mexicano y colega entrañable de Moreno), Director del extinto Instituto de Investigaciones Pedagógicas (1970-1979) y Coordinador del Colegio de Pedagogía (1972-1974).

En gran medida por éste último, hoy permanece vivo gran parte de su legado, a través del vigente Seminario de Pedagogía Universitaria (que él fundó) y la formación de docentes que integran la actual plantilla del Colegio de Pedagogía y otras como la de la Universidad Veracruzana y la Universidad Autónoma de Nuevo León. Asimismo, fue director de 40 tesis, 15 de licenciatura, 18 de maestría y 7 de doctorado. Con un papel distinguido en el diseño, implementación y evaluación de planes y programas de estudio, también organizó exposiciones como la intitulada Infancia, escuela y biblioteca que tuvo lugar en las instalaciones de la Biblioteca Nacional (2003). Antes de fallecer, un 16 de noviembre de 2004 en la Ciudad de México, recibió varios reconocimientos. Entre los más destacados se encuentran el Premio Universidad Nacional (2004), la distinción de Visitante Distinguido por el H. Ayuntamiento de Veracruz (1988) y la Presea Tlacaélel por la Fundación Tlacaélel de Morelos (2001). Todo ello muestra la necesidad de valorar, estudiar y conocer su prolija obra escrita, que fue, sin más, una defensa sui generis de la cultura hispanoparlante frente a la introducción cultural norteamericana a la realidad educativa y pedagógica de la segunda mitad del siglo XX.

\section{Conclusiones}

Enrique Moreno fue testigo de un México de transición entre la apertura a la modernidad (bajo el modelo anglosajón) y la destrucción del legado cultural (cuyo modelo era hispanoamericano). Hijo de dos notables educadores, desde sus primeros años de vida se formó en un ambiente cultural rico, pero con dificultades económicas que pudo superar, en gran medida, por la movilidad social que representó convertirse en investigador, docente y difusor de la cultura. El estatus adquirido fue fruto de una vida orgullosamente universitaria, a la altura del legado de José Vasconcelos. El pensamiento de Moreno respondió, teóricamente, a una crisis educativa y una crisis pedagógica. La primera se debía a un cuestionamiento radical de aquella como institución. La segunda se debía a una interpelación compleja de la pedagogía como ciencia o disciplina. Ambas crisis influyeron en la formación de una personalidad que defendió la tradición pedagógica clásica, en los autores ya mencionados, y censuró epistemológica e históricamente a las Ciencias de la educación, por carecer disciplinariamente de sentido.

Debido a que en su formación se concebía equilibradamente Ciencias y Humanidades, durante su trayectoria profesional combinó exitosamente campos humanísticos (Historia, Estética, Literatura, Arquitectura, etc.) y científicos (Medicina, Filosofía analítica de la ciencia, Antropología, Estadística, etc.). Esto fue algo novedoso para su tiempo, porque conjuntaba, por ejemplo, las unívocas interpretaciones de filósofos analíticos y humanistas clásicos. Su predilección por la pedagogía experimental fue prolífica, pues dio lugar a auténticas invenciones, como aquel original instrumento de educación vocacional. No obstante, esta misma predilección hoy debe ser reinterpretada porque lleva ínsita una inclinación peligrosa al univocismo, el cual resulta difícil de sostener en los debates pedagógicos y educativos recientes. Por mencionar un ejemplo, la hermenéutica ha sido una disciplina que exitosamente se ha cultivado en la pedagogía de las últimas décadas (Flórez, 2005). De esa manera, se advierte la necesidad de contextualizar su postura contra las Ciencias de la educación, pues en otras universidades latinoamericanas, la polémica acontecida en la UNAM se pudo haber vivido de otra forma, incluso sin haber polémica como tal y más bien intercalando, sin problema alguno, los vocablos Pedagogía y Ciencias de la educación (Puiggrós y Marengo, 2013). Lo cierto, es que esta polémica suscitada en la UNAM puso en crisis a la pedagogía como disciplina y pretendió derrumbar la legítima tradición clásica.

En términos de autenticidad, Moreno aportó un planteamiento anti-Ciencias de la educación teóricamente sólido y propuso la teoría pedagógica de los modelos didácticos. Esto es una muestra de la doble originalidad del pensamiento pedagógico mexicano y latinoamericano, primero, para asimilar ideas y tradiciones de pensamiento alemán, francés, inglés y español, y segundo, para crear una explicación 
sistemática sobre el desarrollo de dos grandes formas de educar (una de forma artesanal y otra de forma escolar). Ambas aportaciones revelan una forma de recibir la tradición clásica y actualizar sus ideas en función de problemas propios de la realidad latinoamericana, incluyendo su proyecto editorial nunca más replicado en México. Latinoamérica es epicentro de distintas tradiciones pedagógicas que se expresan en función de las necesidades de cada contexto. En el caso de la tradición mexicana, Moreno propuso una forma de recuperar la tradición propia y evitar asumir ciegamente un pensamiento que no es propio.

De ese modo, la recepción de su pensamiento puede señalar vetas del conocimiento pedagógico que futuras investigaciones pueden explorar. Estas vetas son, al menos, seis: Teoría pedagógica, Epistemología pedagógica, Pedagogía experimental, Orientación educativa, Bibliografía pedagógica e Historia de la educación y la pedagogía. Cuando pedagogos y educadores latinoamericanos se propongan estudiar los diferentes debates del estatuto científico de la pedagogía, será preciso recuperar pensamientos como el de Enrique Moreno y de los Arcos, sea para valorarlo en relación con otros de distintos países, o bien, para actualizarlo de cara a la proyección de una educación adaptada a nuestras circunstancias y una disciplina consciente de su pasado, presente y futuro.

Para ello, será importante dar a conocer su obra más allá de México (a sabiendas de que, incluso, en su propio país sigue siendo desconocido). La difusión de su obra escrita dotará de las necesarias fuentes primarias para su estudio crítico. Finalmente, en la memoria histórica de la pedagogía latinoamericana debería tener un lugar, ya sea por su cuestionamiento de la acrítica recepción de la Ciencias de la educación anglosajonas en el ámbito latinoamericano, y por la rigurosa preservación de la tradición pedagógica clásica que aún espera seguir hermanando países, instituciones educativas y redes de investigación hispanoamericanas.

\section{REFERENCIAS}

Aguirre, M. (1998). Tramas y espejos. Los constructores de historias de la educación. México, México: UNAM.

Bartomeu, M., Juárez, I., Alzueta, S. y Juárez, F. (1993). Epistemología o fantasía. El drama de la pedagogía. México, México: UPN.

Beuchot, M. (2005). Perfiles esenciales de la hermenéutica. México, México: FCE.

Carnoy, M. (1977). La educación como imperialismo cultural. México, México: Siglo XXI.

Delgado, E. (2017). Lenguaje y pedagogía, un olvido en cuestión. Entre notas de Enrique Moreno y de los Arcos y Otto Friedrich Bollnow. En Huarte, R. (Coord.) La filosofia de la educación desde los linderos (pp. 49-64). México, México: Newton/Posgrado UNAM Pedagogía/CONACyT.

Escamilla, J. y Rodríguez, A. (2010). El Método Biográfico en la Investigación Socioeducativa. México, México: UNAM. Flórez, R. (2005). Pedagogía del conocimiento. México, México: McGraw-Hill.

García, P. (2017). Plan de estudios y Curriculum. Una polémica de Enrique Moreno y de los Arcos. Correo del maestro, (254) México, México. Recuperado de https://bit.ly/2Zj2rCG

Illich, I. (1973). ¿En América Latina para qué sirve la escuela? Buenos Aires, Argentina: Búsqueda.

Larroyo, F. (1979). Historia general de la educación. México, México: Porrúa.

Luzuriaga, L. (1980). Historia de la educación y de la pedagogía. Buenos Aires, Argentina: Losada.

Moreno, E. (1993). Principios de pedagogía asistemática. México, México: UNAM.

Moreno, E. (1999). Hacia una teoría pedagógica. México, México: UNAM.

Moreno, E. (2002). Sobre el vocablo pedagogía, Paedagogium. Revista Mexicana de Educación y Desarrollo, (12), México, México (pp. 4-6). Recuperado de https://bit.ly/2EIURuM.

Olivera, A., Rueda, S. y Espejel, L. (coords.) (1998). Historia e historias: cincuenta años de vida académica del Instituto de Investigaciones Históricas. México, México: UNAM.

Peralta, A. (2015). El papel de los clásicos en la pedagogía. Indagaciones en la obra de Enrique Moreno y de los Arcos. El correo del maestro, (182), México, México (pp. 31-40) https://bit.ly/3kRi39j. 
Puiggrós, A. y Marengo, R. (2013). Pedagogía: Reflexiones y debates. Bernal, Argentina: Universidad de Quilmes.

Ursúa, N., Ayestarán, I. y González, J. (2004). Filosofía critica de las ciencias humanas y sociales. México, México: Fontamara.

\section{Notas}

1 Ubicada en la delegación Iztapalapa, al sur de la Ciudad de México. En ese tiempo, todavía la zona no estaba urbanizada y carecía de servicios básicos como la luz y drenaje.

2 Pedagogo y latinista español naturalizado mexicano. Además de ser catedrático, fue un prolijo escritor de obras pedagógicas como Compendio de Ciencia de la educación y las Antologías pedagógicas de Platón, Aristóteles, Quintiliano, San Agustín y Pestalozzi.

3 Pedagogo español. Fue un catedrático que, por su talento y preparación, académica laboró en la UNESCO como investigador y experto consultor en Sudamérica.

4 Normalista y filósofo mexicano. En la UNAM, fue alumno de tres filósofos mexicanos, Antonio Caso (1883-1946), Ezequiel Chávez (1868.1946) y Samuel Ramos (1897-1959) -siendo todos notables catedráticos, los primeros dos fueron rectores y el tercero llegó a ser Secretario de FFyL-. A raíz de una beca conseguida para estudiar en Alemania, introdujo el neokantismo en México. Fue autor de numerosas obras pedagógicas y filosóficas.

5 Pedagogo francés (1918-2016), investigador y docente de la Universidad de Caen. Entre su extensa obra escrita destaca Pedagogía experimental, publicada en FCE (1988).

\section{BY-NC-ND}

